\title{
Evaluating of Loan Guarantees Between Parent and Subsidiary Based on Vulnerable Option
}

Li Li

School of Management and Economics, University of Electronic Science and Technology of China Chengdu, 610054, China

School of Mathematics, Yunnan Normal University

Kunming, 650092, China

\section{Zongfang Zhou}

School of Management and Economics, University of Electronic Science and Technology of China Chengdu, 610054, China

\author{
Xiao Lei \\ Ideological and Political Theory Teaching and Research Department, Kunming University \\ Kunming, 650214, China \\ E-mail: leisurelily@yahoo.cn \\ Received 26 November 2011 \\ Accepted 12 March 2012
}

\begin{abstract}
Loan guarantees between parent and subsidiary is the main related transaction in business group, its correct estimation is the foundation of controlling group's credit risk. Applying option theory, this paper researches the vulnerable option characters of loan guarantees, gives the valuation model under two different security methods, theoretically analyzes the influence of such critical factors as companies asset value and equity ratio to loan guarantees' value and provides theoretical basis for the decision-making of loan guarantees.
\end{abstract}

Keywords: Business group, Vulnerable option, Loan guarantees, Credit risk.

\section{基于脆弱期权的母子公司贷款担保价值研究}

\author{
李丽 ${ }^{1,2}$ 周宗放 ${ }^{1}$ 肖磊 $^{3}$ \\ 1. 电子科技大学/经济与管理学院, 成都 610054 \\ 2. 云南师范大学/数学学院, 昆明 650092 \\ 3. 昆明学院/思政部, 成都 650214
}

\begin{abstract}
摘要：母子公司贷款担保是企业集团主要的一种关联交易行为，对担保价值的正确估算是控制集团整体信 用风险的基础。本文基于期权理论分析了贷款担保的脆弱期权特性，给出了两种不同担保方式下的担保价 值模型, 从理论上分析了母子公司资产价值和股权比例等关键因素对担保价值的影响, 为企业集团母子公 司贷款担保决策提供了理论依据。
\end{abstract}

关键词：企业集团，脆弱期权，贷款担保，信用风险

\section{1. 引言}

近年来, 随着农凯、德隆、铁本等企业集团相 继出现财务危机, 由此而引发的信用危机给企业经 营发展带来的不利影响已经引起集团经营管理者的 注意。一些大型企业集团纷纷成立风险管理部门来 评估各类交易活动带来的风险, 以期将风险控制在
集团整体的风险容忍范围内，从而增强企业自身的 抗风险能力。企业集团在进行外部融资时, 既可以 以母公司作保证人, 由子公司向银行申请贷款, 也 可以以子公司作保证人, 由母公司向银行申请贷 款, 这两种母子公司之间的关联担保行为不仅在企 业集团内母子公司关联交易记录中占有较大比例, 同时其担保的贷款金额占据了商业银行大客户对外 
担保的大部分资金。无论哪一方做保证人, 当借款 人和保证人都不能履行还款责任时, 为维护集团整 体信誉和获得银行长久的信贷支持, 企业集团通常 需要动用内部资本市场上的闲散资金来弥补资金缺 口。如果集团没有足够的风险储备金，担保带来的 风险超出集团整体的风险容忍水平, 关联担保的传 染性、聚集性将会导致企业集团整体发生信用危 机, 给集团的经济发展带来严重后果。然而另一方 面, 过多的风险储备金会降低资源使用效率, 导致 企业集团内部资本市场的投资效率不足, 限制集团 的健康发展。因此, 对母子公司关联担保行为价值 的科学估算和正确认识是衡量贷款担保风险与未来 补偿能力的基础, 有助于加强企业集团信用风险的 管理。

早期关于贷款担保价值的研究文献通常假定保 证人一定具有偿付能力, 即担保本身不存在违约风 险。这是因为在早期的西方发达国家, 保证人一般 是政府或政府机构, 可以看作是无违约风险的担保 人。Merton(1977)首次揭示了无违约风险贷款担保的 看跌期权特性 ${ }^{1}$, 并指出保证人的担保行为类似于承 约看跌期权。利用担保责任与看跌期权之间的同构 关系, Merton 推导出由政府或政府机构提供的贷款 担保价值的解析解。Jones and Mason(1980)拓展了他 的研究工作, 运用连续时间期权定价理论, 对无违 约风险保证人担保的可赎回、附息票债券的担保进 行定价 ${ }^{2}$ 。

随着人们对期权面临的风险的不断认识, 特别 是场外市场交易的期权，其承约方（writer）也有可 能违约, 这类期权被 Johnson 和 Stulz (1987) ${ }^{3}$ 称之 为脆弱期权 (vulnerable option)。类似于看跌期权 承约方的保证人同样也有可能违约。 Lai(1992) ${ }^{4}$ 首次 关注了由私人或私人机构提供担保的贷款担保估值 问题, 他将这种担保称为有违约风险的担保。Lai 采 用离散时间框架下的均衡分析, 将担保价值视为有 担保的贷款价值与无担保的贷款价值之差。孙艳、 郭菊娥等(2008) $)^{5}$ 沿用 Lai 的研究思路, 得到担保物 权未按比例分配下的贷款担保价值。Chang (2006) ${ }^{6}$ 研究了多个担保对象和多个担保企业的担保定价问 题。孙艳, 郭菊娥等人 (2009) ${ }^{7}$ 则在 Chang 的工作 基础上, 建立了担保人与借款人一对多情形下, 存 在优先求偿权的贷款担保定价模型, 侧重剖析了求 偿权的优先次序对担保价值的影响。上述研究都是 以离散时间框架下的均衡分析为基础, 对所得各类 担保价值的比较静态分析采用的是蒙特卡洛模拟方 法。本文将在连续时间框架下, 利用脆弱期权的性 质, 通过严谨的证明得到贷款担保价值的比较静态 分析结论。
另外, 前人的研究都是针对没有股权联系的借 款人与保证人进行的, 所得结论如果直接应用于母 子公司间的贷款担保定价中, 必然会扭曲担保的真 实价值, 不利于控制集团整体信用风险。因此, 有 必要对具有股权联系的母子公司间的担保行为价值 进行科学评估。

本文第二部分首先介绍模型基本假设, 然后分 别建立两种关联担保方式下的贷款担保价值模型, 并利用脆弱期权的性质严格地推导出担保价值的比 较静态分析结论, 重点考察母子公司资产价值、股 权比例、资产相关系数以及贷款金额等重要参数对 担保价值的影响。最后是算例分析。第三部分是本 文的不足和进一步的研究展望。

\section{2. 贷款担保价值模型}

本节将分别建立以母公司作保证人和以子公司 作保证人, 这两种担保方式下的母子公司贷款担保 价值模型, 并作比较静态分析。

\section{1. 模型假设和符号说明}

本文主要分析企业集团内母公司 $P$ 和一个子公 司 $S$ 之间关联担保的情形。假定贷款期限为 $[0, T]$, 到 期还款金额为 $D$, 为简化问题起见, 在贷款期内不 考虑两公司各自原有负债。沿用文献 8 中的假设, 在任意的 $t \in[0, T]$ 时刻, 母公司 $P$ 和子公司 $S$ 的资产 价值分别记为 $P(t)$ 和 $S(t)$, 且 $P(t)$ 和 $S(t)$ 服从二维几 何布朗运动, 即:

$$
\left[\begin{array}{l}
d \ln (S(t)) \\
d \ln (V(t))
\end{array}\right]=\left[\begin{array}{l}
\mu_{S} \\
\mu_{P}
\end{array}\right] d t+\Omega\left[\begin{array}{l}
d W_{1}(t) \\
d W_{2}(t)
\end{array}\right]
$$

其中, $\mu_{S} 、 \mu_{P}$ 是常数, 表示资产价值漂移 率, $W_{1}(t)$ 和 $W_{2}(t)$ 是独立的布朗过程, $\Omega$ 是常数二 阶矩阵, 满足

$$
\Omega \Omega^{\prime}=\left[\begin{array}{cc}
\sigma_{S}^{2} & \rho_{P S} \sigma_{S} \sigma_{P} \\
\rho_{P S} \sigma_{S} \sigma_{P} & \sigma_{P}^{2}
\end{array}\right]
$$

$\sigma_{P}, \sigma_{S}$ 分别表示母子公司资产价值波动率, $\rho_{P S}$ 是 它们之间的相关系数。

根据文献8的分析，母子公司资产价值关系可以 表示为 $P(t)=Y(t)+\lambda S(t)$ 。这里, $Y(t)$ 示母公司 $P$ 的 总价值减去所含子公司 $S$ 的那部分价值后剩下的价 值。 $\lambda$ 表示母公司 $P$ 持有子公司 $S$ 的股权比例。 
令 $\sigma_{Y}^{2}=\operatorname{Var}(Y(t)), \quad \rho_{Y S}=\frac{\operatorname{Cov}(Y(t), S(t))}{\sigma_{Y} \sigma_{S}}$ 表示母公司 总价值中不包含子公司的那部分价值 $Y(t)$ 与其子公司 资产价值 $S(t)$ 变化的相关系数, 则有

$$
\rho_{P S}=\frac{\sigma_{Y}}{\sigma_{P}} \rho_{Y S}+\lambda \frac{\sigma_{S}}{\sigma_{P}}
$$

上式表明, 当固定 $\lambda 、 \sigma_{P} 、 \sigma_{S}$ 与 $\sigma_{Y}$ 时, $\rho_{P S}$ 与 $\rho_{Y S}$ 成正比, 记作: $\rho_{P S} \propto \rho_{Y S}$ 。这表示在其它条 件不变的情况下, $Y(t)$ 部分资产与子公司资产价值相 关性越强, 母子公司资产价值相关性越强; 类似 地, 当固定 $\sigma_{P} 、 \sigma_{S} 、 \sigma_{Y}$ 与 $\rho_{Y S}$ 时, $\rho_{P S}$ 与 $\lambda$ 成正 比, 记作: $\rho_{P S} \propto \lambda$ 。表示当其它条件不变时, 母公 司对子公司的持股比例越大, 母子公司资产价值相 关性越强。

\section{2. 贷款担保价值}

\subsection{1 母公司 $P$ 为子公司 $S$ 贷款提供担保情形}

假设子公司 $S$ 向银行申请到期还款金额为 $D$ 的 贷款, 母公司 $P$ 为这笔贷款提供担保。在贷款期末 $T$ 时刻, 考虑到保证人也有可能不能足额偿付剩余 贷款, 因此, 根据母子公司资产价值情况, 可以得 到母公司作为保证人所需提供的支付具有以下三种 情形:

(1) 子公司的资产 $S(T)$ 足以支付贷款 $D$ 时, 母公 司无需承担连带担保责任, 支付为 0 。

(2) 子公司的资产 $S(T)$ 不足以支付贷款 $D$, 母公 司的剩余资产 $Y(T)$ 足以偿付贷款余额 $D-S(T)$, 此时 母公司因为担保, 所需提供的支付为 $D-S(T)$ 。

(3) 子公司的资产 $S(T)$ 不足以支付贷款 $D$, 母公 司的剩余资产 $Y(T)$ 也不足以偿付贷款余额 $D-S(T)$, 此时母公司因为连带担保责任, 将其所有剩余资产 支付给银行, 提供的支付为 $Y(T)$ 。

综上所述, 母公司因为提供担保, 在 $T$ 时刻所需 提供的支付结构为:

$$
\begin{aligned}
& L^{P S}(T)=\left\{\begin{array}{ccc}
0, & \text { if } \quad S(T) \geq D \\
D-S(T), & \text { if } \quad S(T)<D \text { and } Y(T) \geq D-S(T) \\
Y(T), & \text { if } \quad S(T)<D \text { and } Y(T)<D-S(T)
\end{array}\right. \\
& =\min [Y(T), \quad \max (D-S(T), \quad 0)] \\
& =\min [P(T)-\lambda S(T), \quad \max (D-S(T), \quad 0)]
\end{aligned}
$$

由以上支付结构可知, 母公司为子公司的贷款 $D$ 提供担保相当于以母公司做承约人, 出售一份以
子公司资产为标的、执行价格为贷款金额的欧式脆 弱看跌期权。因此, 贷款的担保价值为欧式脆弱看 跌期权价值。

根据Johson ${ }^{3}$, 可知贷款担保价值 $L^{P S}$ 满足边界条 件为 (4) 式的下列微分方程

$$
\begin{aligned}
& r x-r P \frac{\partial x}{\partial P}-r S \frac{\partial x}{\partial S}-\frac{1}{2} \sigma_{S}^{2} S^{2} \frac{\partial^{2} x}{\partial S^{2}} \\
& -\frac{1}{2} \sigma_{P}^{2} P^{2} \frac{\partial^{2} x}{\partial P^{2}}-\rho_{P S} \sigma_{P} \sigma_{S} \frac{\partial^{2} x}{\partial P \partial S}=-\frac{\partial x}{\partial T}
\end{aligned}
$$

在风险中性假设下, 贷款担保的价值为

$$
\begin{aligned}
& L^{P S}=E^{Q}\left[e^{-r T} L^{P S}(T)\right] \\
& =e^{-r T} E^{Q}\{\min [P(T)-\lambda S(T), \quad \max (D-S(T), \quad 0)]\}
\end{aligned}
$$

其中 $E^{Q}[$.$] 表示在风险中性概率空间下求期望，$ $r$ 是无风险利率。

\subsection{2 子公司 $S$ 为母公司 $P$ 贷款提供担保情形}

同上节分析方法类似，当子公司 $S$ 为母公司 $P$ 的贷款提供担保责任时，考虑到子公司也有可能不 能足额偿付剩余贷款，因此根据期末母子公司资产 价值情况, 可以得到子公司作为保证人所需提供的 支付结构为

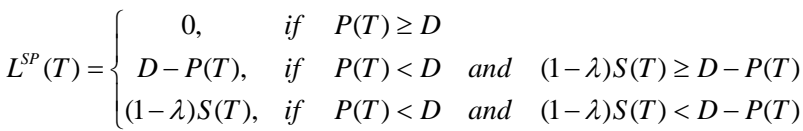

$$
\begin{aligned}
& =\min [(1-\lambda) S(T), \quad \max (D-P(T), \quad 0)]
\end{aligned}
$$

同理根据 Johson ${ }^{3}$, 可知贷款担保价值 $L^{S P}$ 满足边 界条件为（7）式的如（5）式的微分方程。

在风险中性假设下，贷款担保的价值为

$$
\begin{aligned}
& L^{S P}=E^{Q}\left[e^{-r T} L^{S P}(T)\right] \\
& =e^{-r T} E^{Q}\{\min [(1-\lambda) S(T), \quad \max (D-P(T), \quad 0)]\}
\end{aligned}
$$

\section{3. 比较静态分析}

引理：记 $p(S(t), V(t), X, T)$ 为欧式脆弱看跌期权在 $t$ 时刻的价值, 其中 $S(t)$ 表示标的资产价值, $V(t)$ 表 示期权承约方资产价值, $X$ 为执行价格, $T$ 为到期 日，则有以下三式成立:

$$
\frac{\partial p}{\partial S}<0 \quad, \quad \frac{\partial p}{\partial V}>0 \quad, \quad \frac{\partial p}{\partial X}>0
$$

证明：易知 $p(S(t), V(t), X, T)$ 在到期时的支付为 $\min [V(T), \max (X-S(T), 0)]$ 。构造一人造资产 $a(t)$, 
满足条件 $a(T)=\max (S(T), \quad X-V(T))$, 则有下式成 立

$\max (X-a(T), \quad 0)=\min (V(T), \quad \max (X-S(T), \quad 0))$ 即以 $a(t)$ 为标的资产的普通欧式看跌期权 $p^{a}(a(t), X, T)$ 与脆弱看跌期权 $p(S(t), V(t), X, T)$ 具有 相同的价值。令 $e(x, y, T)$ 表示在 $T$ 时刻以价值为 $y$ 的资 产交换价值为 $x$ 的资产的欧式期权的现值。由 Margrabe $^{9}$, 有

$$
\begin{aligned}
& a(t)=S(t)+e[X \exp (-r(T-t))-V(t), \quad S(t), \quad T] \\
& =S(t)+e^{I} \\
& =S(t)+X \exp (-r(T-t))-V(t)-S(t) \\
& +e[S(t), \quad X \exp (-r(T-t))-V(t), \quad T] \\
& =X \exp (-r(T-t))-V(t)+ \\
& e[S(t), \quad X \exp (-r(T-t))-V(t), \quad T] \\
& =X \exp (-r(T-t))-V(t)+e^{I I}
\end{aligned}
$$

$$
\text { 上式表明 } \frac{\partial a}{\partial S}=e_{1}^{I I}>0, \frac{\partial a}{\partial V}=-e_{1}^{I}<0 ，
$$
$\frac{\partial a}{\partial X}=e^{-r(T-t)} e_{1}^{I}>0$ 。其中, 下标 1 表示对第一个分量 取偏导数。进一步, 由 Merton $^{10}$ 中普通欧式看跌期 权的性质有:

$$
\begin{aligned}
& \frac{\partial p}{\partial S}=p_{1}^{a} \frac{\partial a}{\partial S}<0, \frac{\partial p}{\partial V}=p_{1}^{a} \frac{\partial a}{\partial V}>0, \\
& \frac{\partial p}{\partial X}=p_{1}^{a} \frac{\partial a}{\partial X}>0 。
\end{aligned}
$$

证毕

引理表明欧式脆弱看跌期权的价值是关于标的 资产价值递减, 关于承约方资产价值和执行价格递 增的函数。

结论 1 : 当母公司为子公司贷款提供担保时, 贷款担保价值关于子公司资产价值和母公司对子公 司的持股比例单调递减, 关于母公司资产价值和贷 款金额单调递增。

证明：令 $H=\max (D-S(T), 0)$, 由引理可知,

$$
\begin{aligned}
\frac{\partial L^{P S}}{\partial S} & =L_{1}^{P S} \frac{\partial Y}{\partial S}+L_{2}^{P S} \frac{\partial H}{\partial S}=(-\lambda) L_{1}^{P S}+L_{2}^{P S} \frac{\partial H}{\partial S}<0 \\
\frac{\partial L^{P S}}{\partial P} & =L_{1}^{P S}>0 \\
\frac{\partial L^{P S}}{\partial D} & =L_{2}^{P S} \frac{\partial H}{\partial D}>0 \\
\frac{\partial L^{P S}}{\partial \lambda} & =(-S) L_{1}^{P S}<0
\end{aligned}
$$

下标 $1 、 2$ 分别表示对第 $1 、 2$ 个分量取偏导数。 这表明, 当母公司为子公司贷款提供担保时, 在其他条件不变的情况下, 子公司资产价值越大, 贷款担保价值越低; 母公司资产价值越大，贷款担 保价值越高; 贷款金额越大，贷款担保价值越高; 母公司对子公司的持股比例越小，贷款担保价值越 高。

类似地可以证明, 当子公司为母公司贷款提供 担保时, 有下列各式成立

$$
\frac{\partial L^{S P}}{\partial S}>0, \frac{\partial L^{S P}}{\partial P}<0, \frac{\partial L^{S P}}{\partial D}>0, \frac{\partial L^{S P}}{\partial \lambda}<0
$$

于是有结论 2：当子公司为母公司贷款提供担 保时, 贷款担保价值关于母公司资产价值和母公司 对子公司的持股比例单调递减，关于子公司资产价 值和贷款金额单调递增。

这表明当子公司为母公司贷款提供担保时, 在 其他条件不变的情况下, 母公司资产价值越大, 贷 款担保价值越低; 子公司资产价值越大，贷款担保 价值越高; 贷款金额越大，贷款担保价值越高; 母 公司对子公司的持股比例越小, 贷款担保价值越 高。

综合以上分析发现, 无论是母公司做保证人还 是子公司做保证人，借款人资产价值越低，贷款担 保价值越高; 保证人资产价值越高, 贷款担保价值 越高; 贷款金额越大，担保越有价值。这符合人们 的经济直觉。另外, 母公司对子公司持股比例越 小，保证人的支付越大，贷款担保价值就越高。

注意到 $\rho_{P S} \propto \lambda$, 因此母子公司资产价值相关性 越弱, 贷款担保价值越高。这是因为, 相关性越弱 说明母子公司之间分散风险的能力越强, 担保越有 价值。

另一方面, 如果母公司与子公司分别处于行业 互补型企业，且剥离子公司资产后的母公司剩余资 产 $Y$ 与子公司资产 $S$ 负相关。由 $\rho_{P S} \propto \rho_{Y S}$ 知, 这种 关系下的贷款担保价值要高于同行业内母子公司之 间的贷款担保价值。换句话说, 行业互补型母子公 司关联担保给集团控制整体信用风险带来的压力要 小于同行业内母子公司关联担保, 原因在于行业互 补有助于风险的分散。

\section{4. 算例分析}

假设某集团母公司资产初值为 $P(0)=4000$ (单 位: 万元）, 旗下一子公司资产初值为 $S(0)=2400$ （单位：万元），母公司对子公司的持股比例为 $\lambda=0.7$, 子公司于 2008 年初通过由母公司作担保从 银行获得一笔贷款, 承诺在2011年初归还贷款的面 值为 $D=2000$ (单位: 万元)。其余参数取值同参考 
文献 8 , 具体如下: $\mu_{P}=0.05, \sigma_{P}=0.4$, $\mu_{S}=0.05, \sigma_{S}=0.35, \sigma_{Y}=0.2 ， \rho_{P S}=0.8 ，$ $r=5 \%$ 。将参数代入 (6) 式, 模拟 50000 次, 计算得 到 $L^{P S}=163.53$ (万元) , 即这笔贷款的担保价值为 163.53 万。如果集团内闲散资金多于 163.53 万，那么 这种担保行为不会增加集团整体的信用风险。

假设这笔贷款是由母公司向银行申请, 而子公 司做保证人, 其余参数同上, 代入 (8) 式, 模拟计 算可得 $L^{S P}=44.51$ (万元)。如果风险管理部门认 为, 集团的风险承受能力或者能够承受的最大风险 损失为 100 万, 那么根据上述计算结果, 建议企业 集团进行外部融资时, 由子公司作保证人, 以母公 司名义向银行申请贷款, 则可以降低集团整体的信 用风险。

\section{3. 小结}

本文应用期权理论分析了母子公司贷款担保的 脆弱期权特性, 给出了以母公司作保证人和以子公 司作保证人, 这两种不同担保方式下的担保价值模 型, 从理论上分析了母子公司资产价值、股权比 例、资产价值相关性等关键因素对担保价值的影 响。结果表明, 无论是母公司作保证人, 还是子公 司作保证人，保证人资产价值以及贷款金额的增大 都会增加担保价值, 借款人资产价值的增大会降低 担保价值; 在其他因素不变的情况下, 母公司对子 公司持股比例的减少会增加保证人的支付, 进而增 大担保价值; 同时, 母子公司资产价值相关性越 弱, 贷款的担保价值就越高。特别是从控制风险角 度来看, 行业互补型母子公司关联担保给集团控制 整体信用风险带来的压力要小于同行业内母子公司 关联担保。算例分析发现, 即使向银行申请同样金 额的贷款, 在不同的担保方式下, 贷款的担保价值 不同。因此, 企业集团可以选择合适的担保方式, 以降低集团整体的信用风险。

集团风险管理者在评估担保行为价值时，还应 注意资产波动对担保价值的影响, 但是标的资产和 承约方资产波动对脆弱期权价值的影响较为复杂, 本文未能从理论上得出母子公司资产波动对担保价 值的影响, 这将是我们要进一步深入研究的问题。

\section{致谢}

本文感谢下列基金的资助：国家自然科学基金 项目（70971015,11061041）; 云南省教育厅科学研 究基金项目（2011C109）。
1. R. C. Merton, An Analytic Derivation of the Cost of Loan Guarantees and Deposit Insurance: An Application of Modern Option Pricing Theory, Journal of Banking and Finance. (6) (1977) 3-11.

2. E. P. Jones and S.P. Mason, The Valuation of Loan Guarantees, Journal of Banking and Finance. 4(1980) 89-107.

3. H. Johnson and R. Stulz, The Pricing of Options with Default Risk, Journal of Finance. (42) (1987) 267-280.

4. V. S. Lai, An Analysis of Private Loan Guarantee, Journal of Financial Services Research. 6(1992) 223248.

5. Y. Sun etc, The Research on the Value of the Loan Guarantee under Security Interest in No Proportion, Forecasting. 27(2) (2008) 73-76. 孙艳, 郭菊娥,王乐,曹华, 担保物权未按比例分配 的贷款担保价值研究, 预测. 27(2) (2008)73-76.

6. C. C. Chuang, S. L. Chung and M. T. Yu, Loan Guarantee Portfolios and Joint Loan Guarantees with Stochastic Interest Rates, The Quarterly Review of Economics and Finance. (46) (2006) 16-35.

7. Y. Sun etc, Research on the Loan Guarantee Value with the Claim Priority, Management Review. 21(4) (2009) 34-41.

孙艳, 郭菊娥,王乐,曹华, 优先求偿权条件下贷款 担保价值研究, 管理评论, 21(4) (2009) 34-41.

8. L. Chen and Z. F. Zhou, A Research Based Shareholding Ratio on Default Contagion between Parent and Subsidiary Company in an Enterprise Group, Journal of Industrial Engineering and Engineering Management. 3(2009) 80-84.

陈林,周宗放, 基于股权比重的企业集团内母子公 司之间信用风险传递研究，管理工程学报. 3(2009) 80-84.

9. W. Margrabe, The Value of an Option to Exchange One Asset for Another, Journal of Finance. 33(1978) 177186.

10. R. C. Merton, The Theory of Rational Option Pricing, Journal of Economics and Management Science. 4(1973) 141-183.

\section{参考文献}

